\title{
A MULTI-SORTED VERSION OF SECOND ORDER ARITHMETIC
}

\author{
FARIDA KACHAPOVA
}

\begin{abstract}
This paper describes axiomatic theories $S A$ and $S A R$, which are versions of second order arithmetic with countably many sorts for sets of natural numbers. The theories are intended to be applied in reverse mathematics because their multi-sorted language allows to express some mathematical statements in more natural form than in the standard second order arithmetic. We study metamathematical properties of the theories $S A, S A R$ and their fragments. We show that $S A$ is mutually interpretable with the theory of arithmetical truth $P A T r$ obtained from the Peano arithmetic by adding infinitely many truth predicates. Corresponding fragments of $S A$ and $P A T r$ are also mutually interpretable. We compare the proof-theoretical strengths of the fragments; in particular, we show that each fragment $S A_{s}$ with sorts $\leqslant s$ is weaker than next fragment $S A_{s+1}$.
\end{abstract}

\section{INTRODUCTION}

Reverse mathematics is one of the current research directions in mathematical logic. It is based on the formal theories, subsystems of second order arithmetic, introduced by Simpson [4]. Here we describe two other versions of second order arithmetic that can possibly be used in reverse mathematics. They are axiomatic theories $S A$ and $S A R$. Both theories have countably many sorts for sets of natural numbers, a predicative comprehension axiom and a version of choice axiom. The difference between the two systems is in the induction axiom: $S A$ has the full induction and $S A R$ has the induction restricted to formulas of the form $n \in x$.

We believe that in the multi-sorted language of $S A$ and $S A R$ some mathematical statements can be expressed in a shorter and more natural way than in the language of the standard second order arithmetic, which has only one sort for sets of natural numbers. In this paper we study metamathematical

Date: September 3, 2016.

2010 Mathematics Subject Classification. 03B30 Foundations of classical theories (including reverse mathematics); 03F25 Relative consistency and interpretations.

Key words and phrases. Second-order arithmetic; reverse mathematics; predicative; interpretation; relative consistency; truth predicate; proof-theoretical strength.

Australasian Journal of Logic (13:5) 2016, Article no. 3 
properties of $S A, S A R$ and their fragments. We construct an interpretation of $S A$ in the theory of arithmetical truth $P A T r$ introduced in [1. We also construct an interpretation of $P A T r$ in $S A$. Thus, we show that $S A$ and $P A T r$ are equiconsistent. We also prove equiconsistency of fragment $S A_{s}$ of $S A$ with sorts $\leqslant s$ and corresponding fragment $\operatorname{PATr}_{s}$ of $\operatorname{PATr}(s \geqslant 0)$. We show that each fragment $S A_{s}$ is proof-theoretically weaker than next fragment $S A_{s+1}(s \geqslant 0)$. The same is true for fragments of PATr.

In section 2 we give definitions of the axiomatic theories $S A, S A R$ and $P A T r$. In section 3 we explain the motivation for introducing and studying these theories. In section 4 we construct an interpretation of $S A$ in $P A T r$. In section 5 we construct an interpretation of $P A T r$ in $S A$. In section 6 we compare the proof-theoretical strengths of fragments of $S A$ and $P A T r$.

In the rest of the introduction we explain some notations and terminology.

All theories considered in this paper are first-order axiomatic theories (a wellknown definition of a first-order axiomatic theory can be found, for example, in [3]).

The symbol $\leftrightharpoons$ means "equals by definition". The symbol $\diamond$ denotes a logical connective $\wedge, \vee$ or $\supset$, and the symbol $Q$ denotes a quantifier $\forall$ or $\exists$. In each of our axiomatic theories we have the logical constant $\perp$ for falsity and we regard $\neg \varphi$ as an abbreviation for $\varphi \supset \perp$. The complexity of a formula $\varphi$ is the number of occurrences of logical symbols (the main three connectives and quantifiers) in $\varphi$. For any formula $\varphi$ we denote $\overline{\bar{\varphi}}$ the closure of $\varphi$, that is, the formula $\varphi$ with universal quantifiers over all its parameters. We denote $\tau\left[x_{1}, \ldots, x_{n} / t_{1}, \ldots, t_{n}\right]$ the result of proper substitution of terms $t_{1}, \ldots, t_{n}$ for variables $x_{1}, \ldots, x_{n}$ in an expression $\tau$. The complexity of a term $t$ is the number of occurrences of functional symbols in $t$.

We fix a one-to-one coding of all finite sequences of natural numbers such that 0 is the code for the empty sequence. In a theory containing first-order arithmetic we use the notations:

$\left(n_{1}, \ldots, n_{k}\right)$ as the code for sequence $n_{1}, \ldots, n_{k}$;

$(n)_{i}$ for the $i$ th element of the sequence with code $n$;

$l h(n)$ for the length of the sequence with code $n$.

In particular, $(m, n)$ is the code for pair $m, n$ of natural numbers. For a natural number $n$ we denote $\bar{n}$ the formal arithmetical term for $n$, that is $\bar{n}=$ $\underbrace{1+1 \ldots+1}_{n \text { times }}$.

We assume that for any axiomatic theory $K$ some Gödel numbering of its expressions is fixed. For an expression $q$ we denote $\llcorner q\lrcorner$ the Gödel number of $q$

Australasian Journal of Logic (13:5) 2016, Article no. 3 
in this numbering; $t_{m}$ and $\varphi_{m}$ denote the term and formula with Gödel number $m$, respectively.

The notation $K \vdash \varphi$ means that formula $\varphi$ is derivable in theory $K$. The theory $K$ is consistent if it is not true that $K \vdash \perp$. Proof $f_{K}(m, n)$ denotes the arithmetical formula stating that $n$ is the Gödel number of a formal proof in the theory $K$ for formula $\varphi_{m}$. The formula $P v_{K}(m) \leftrightharpoons \exists n P r o o f_{K}(m, n)$ means that $\varphi_{m}$ is derivable in the theory $K$. The formula $\operatorname{Con}_{K} \leftrightharpoons \neg P v_{K}(\llcorner\perp\lrcorner)$ means that the theory $K$ is consistent. Thus, a theory $A$ is proof-theoretically weaker than a theory $B$ (equivalently, $B$ is proof-theoretically stronger than $A$ ) if $B \vdash \operatorname{Con}_{A}$.

In this paper we consider axiomatic theories where variables have superscripts for sorts. A superscript for a variable is usually omitted when the variable is used for the second time or more in a formula or in a proof (so its sort is obvious).

\section{Definition of three AXiomatic theories}

2.1. Axiomatic theory $S A$. $S A$ stands for arithmetic with sorts. The language of theory $S A$ has the following variables:

$n_{1}, n_{2}, \ldots, m, n, \ldots$ over natural numbers and

$x_{1}^{(k)}, x_{2}^{(k)}, \ldots, x^{(k)}, y^{(k)}, \ldots$ of sort $k$ over sets of natural numbers $(k=$ $1,2, \ldots)$.

The language of $S A$ has two numerical constants 0 and 1, and functional symbols $\cdot$ and + . There are the following predicate symbols:

$=$ (equality of natural numbers) and $\epsilon_{k}(k=1,2, \ldots)$.

Numerical terms are constructed from numerical variables and constants using functional symbols. Atomic formulas are:

$t=\tau ; t \in_{k} x^{(k)}$, where $t$ and $\tau$ are numerical terms.

Formulas are constructed from atomic formulas and $\perp$ using logical connectives and quantifiers. The language of $S A$ is defined.

A formula $\varphi$ of $S A$ is called $k$-simple if it has no quantifiers over set variables and it has no variables of sorts greater than $k$.

Equality of sets is introduced as an abbreviation:

$$
x^{(k)}={ }_{k} y^{(k)} \leftrightharpoons \forall n\left(n \in_{k} x \equiv n \in_{k} y\right) .
$$

For brevity we will often omit indices in $=_{k}$ and $\epsilon_{k}$.

Axiomatic theory $S A$ has the following axioms.

1. Classical predicate logic with equality.

2. Peano axioms.

$$
\begin{aligned}
& \neg(n+1=0) . \\
& n+1=m+1 \supset n=m .
\end{aligned}
$$

Australasian Journal of Logic (13:5) 2016, Article no. 3 


$$
\begin{aligned}
& n+0=n . \\
& n+(m+1)=(n+m)+1 . \\
& n \cdot 0=0 . \\
& n \cdot(m+1)=n \cdot m+n .
\end{aligned}
$$

3. Induction axiom. $\varphi(0) \wedge \forall n[\varphi(n) \supset \varphi(n+1)] \supset \forall n \varphi(n)$, where $\varphi$ is any formula of $S A$.

4. Comprehension axiom. $\exists z^{(k)} \forall n(n \in z \equiv \varphi(n))$, where $\varphi$ is a $k$-simple formula not containing the variable $z^{(k)}$.

5. Choice axiom.

$$
\forall n \exists ! x^{(k)} \varphi(n, x) \supset \exists y^{(k+1)} \forall n \exists x^{(k)}[\varphi(n, x) \wedge \forall m(m \in x \equiv(n, m) \in y)],
$$

where $\varphi$ is a $k$-simple formula.

This completes the definition of the theory $S A$. For $s \geqslant 0$ we denote $S A_{s}$ the fragment of $S A$ containing only sorts not greater than $s$. Thus, $S A_{0}$ is the Peano arithmetic $P A$.

2.2. Axiomatic theory $S A R$. $S A R$ stands for arithmetic with sorts and restricted induction. $S A R$ has the same languge as $S A$ and the same axioms, except the induction axiom, which in $S A R$ has the following form:

$$
0 \in x^{(k)} \wedge \forall n\left[n \in x^{(k)} \supset n+1 \in x^{(k)}\right] \supset \forall n\left(n \in x^{(k)}\right), k=1,2, \ldots
$$

For $s \geqslant 0$ we denote $S A R_{s}$ the fragment of $S A R$ containing only sorts not greater than $s$. We consider the theory $S A R$ because its induction axiom is the same as in most of the Simpson's theories [4].

2.3. Axiomatic theory PATr. PATr stands for Peano arithmetic with truth predicates. This theory was introduced in [1. Theory $P A T r$ is based on the axiomatic theory $P A$ for the first-order arithmetic. The language of $P A T r$ is obtained from the language of $P A$ by adding predicate symbols $\operatorname{Tr}_{k}(m, l), k=$ $1,2, \ldots$.

For any $s \geqslant 1$, the language $P A T r_{s}$ is obtained from the language of $P A$ by adding predicate symbols $\operatorname{Tr}_{k}(m, l), 1 \leqslant k \leqslant s$. The language $P A T r_{0}$ is just the language of $P A$.

Let us fix Gödel numbering of expressions of the language PATr. It will be clear from context whether we use Gödel numbering for expressions of PATr or $S A$. Next we introduce some arithmetical formulas.

$\operatorname{Form}(k, m) \leftrightharpoons$ " $m$ is the Gödel number of a formula of $\operatorname{PATr}_{k}$ ".

$\operatorname{Subform}(m, r) \leftrightharpoons$ " $r$ is the Gödel number of a subformula of the formula with Gödel number $m$ ".

$\operatorname{Param}(m, i) \leftrightharpoons " n_{i}$ is a parameter of the expression of $P A T r$ with Gödel number $m "$. 
The following formula means that a sequence $l$ is an evaluation of all parameters of the expression with Gödel number $m$ :

$E v(m, l) \leftrightharpoons(\forall i \leqslant m)[\operatorname{Param}(m, i) \supset l h(l) \geqslant i]$

This formula says that the sequence $l$ is long enough to contains a value for each parameter of the expression with Gödel number $m$ (the intended value for variable $n_{i}$ is the $i$-th element of the sequence $l$ ).

We denote eval and subst the primitive recursive functions such that:

$\operatorname{eval}(m, l)$ equals the value of term $t_{m}$ under evaluation $l$;

$\operatorname{subst}(l, i, n)$ equals the evaluation $l$, in which the $i$-th element is substituted by $n$.

Axiomatic theory PATr has classical predicate logic with equality and the following non-logical axioms.

1. Peano axioms (the same as in $S A$ ).

2. Induction axiom. $\varphi(0) \wedge \forall n[\varphi(n) \supset \varphi(n+1)] \supset \forall n \varphi(n)$, where $\varphi$ is any formula of $P A T r$.

3. Axioms for truth predicates (for any $k \geqslant 1$ ).

(Tr1) $\operatorname{Tr}_{k}(m, l) \supset \operatorname{Form}(\overline{k-1}, m) \wedge E v(m, l)$;

$(\operatorname{Tr} 2) E v(m, l) \wedge " \varphi_{m}$ is $t_{i}=t_{j} " \supset\left[\operatorname{Tr}_{k}(m, l) \equiv(\operatorname{eval}(i, l)=\operatorname{eval}(j, l))\right]$;

$(\operatorname{Tr} 3) E v(m, l) \wedge " \varphi_{m}$ is $\operatorname{Tr}_{k}\left(t_{i}, t_{j}\right) "$

$$
\supset\left[\operatorname{Tr}_{k+1}(m, l) \equiv \operatorname{Tr}_{k}(\operatorname{eval}(i, l), \operatorname{eval}(j, l))\right] ;
$$

$(\operatorname{Tr} 4) \neg \operatorname{Tr}_{k}(\llcorner\perp\lrcorner, l)$;

$(\operatorname{Tr} 5) E v(m, l) \wedge " \varphi_{m}$ is $\varphi_{i} \diamond \varphi_{j} " \supset\left[\operatorname{Tr}_{k}(m, l) \equiv\left(\operatorname{Tr}_{k}(i, l) \diamond \operatorname{Tr}_{k}(j, l)\right)\right]$;

(Tr6) $E v(m, l) \wedge ~ " \varphi_{m}$ is $Q n_{i} \varphi_{j} " \supset\left[\operatorname{Tr}_{k}(m, l) \equiv \operatorname{QnTr}_{k}(j, \operatorname{subst}(l, i, n))\right]$.

The axioms $(\operatorname{Tr} 1)-(\operatorname{Tr} 6)$ describe $T r_{k}$ as the truth predicate for formulas of the language $P A T r_{k-1}$; that is, $\operatorname{Tr}_{k}(m, l)$ means that the formula $\varphi_{m}$ is true under evaluation $l$.

This completes the definition of the theory $P A T r$. Denote $P A T r_{s}$ the fragment of $P A T r$ in the language $P A T r_{s}$. Clearly, $P A T r_{0}$ is just the first-order arithmetic $P A$.

The following lemma describes some properties of the theories $P A T r_{s}$.

Lemma 2.1. Suppose $1 \leqslant q \leqslant s$.

1. If $\psi$ is an arbitrary formula of $P A T r_{q-1}$ with all its parameters in the list $n_{1}, \ldots, n_{k}$, then

$$
P A T r_{s} \vdash l h(l) \geqslant \bar{k} \supset\left(\operatorname{Tr}_{q}(\llcorner\psi\lrcorner, l) \equiv \psi\left[n_{1}, \ldots, n_{k} /(l)_{1}, \ldots,(l)_{\bar{k}}\right]\right) .
$$

Australasian Journal of Logic (13:5) 2016, Article no. 3 
2. $\begin{array}{r}P A T r_{s+1} \vdash 1 \leqslant q \leqslant \bar{s} \wedge \operatorname{Form}(q-1, m) \wedge \forall i[\operatorname{Param}(m, i) \supset i \leqslant r] \supset \\ P v_{P A T R_{s}}\left(\left\llcorner l h(l) \geqslant r \supset\left\{\operatorname{Tr}_{q}(m, l) \equiv \varphi_{m}\left[n_{1}, \ldots, n_{r} /(l)_{1}, \ldots,(l)_{r}\right]\right\}\right\lrcorner\right) .\end{array}$

Proof. 1. Proof is by induction on the complexity of $\psi$.

2. This is proven by formalizing the proof of part 1 in $P A T r_{s+1}$.

\section{More about the three axiomatic theories}

Simpson [4] described and studied subsystems of second order arithmetic that are widely used in reverse mathematics. The main five of these theories are $R C A_{0}, W K L_{0}, A C A_{0}, A T R_{0}$ and $\Pi_{1}^{1}-C A_{0}$, in order of their strenghts.

We introduce the theories $S A$ and $S A R$ as other possible axiomatic theories for reverse mathematics. The theory $S A R_{1}$ is the same as $A C A_{0}$ and the theory $S A_{1}$ is the same as $A C A$ ( $A C A_{0}$ with full induction). Ordinary mathematics can be developed in $S A$ in a similar way that Simpson [4] develops it in the theory $A C A_{0}$. We believe that some mathematical definitions and statements can be simplified in $S A$ due to its multi-sorted language but this requires more research. In this paper we study some metamathematical properties of the theory $S A$.

In sections 4 and 5 we show that the theories $S A$ and $P A T r$ are mutually interpretable, and so are their corresponding fragments. The theory PATr was introduced to clarify the meaning of different sorts of sets (they are defined by formulas in the predicative comprehension axiom); it is also used in the proof of Theorem 5.2 .

\section{Interpretation OF $S A$ In $P A T r$}

We want to represent each set of sort $k$ from $S A$ by a formula of $P A T r_{k-1}$. In the introduction we mentioned Gödel numbering and related notations. In this section we fix Gödel numbering of all expressions of the language PATr. For an expression $q$ we denote $\llcorner q\lrcorner$ the Gödel number of $q$ in this numbering; $\varphi_{r}$ denotes the formula with Gödel number $r$ and $\left\llcorner n_{i} \cdot \varphi_{r}\right\lrcorner$ denotes the Gödel number of the expression $n_{i} \cdot \varphi_{r}$.

We define an arithmetical formula $S t$ by the following:

$$
\begin{aligned}
& S t(k, m) \leftrightharpoons k \geqslant 1 \wedge(\exists i \leqslant m)(\exists r \leqslant m)(\exists l \leqslant m)[\operatorname{Form}(k-1, r) \\
&\left.\wedge m=\left(k,\left\llcorner n_{i} \cdot \varphi_{r}\right\lrcorner, l\right) \wedge E v\left(\left\llcorner\forall n_{i} \varphi_{r}\right\lrcorner, l\right)\right] .
\end{aligned}
$$

Here $\varphi_{r}$ is a formula of the language $P A T r_{k-1}$ and each parameter of $\varphi_{r}$, except $n_{i}$, is evaluated by a sequence $l$. So the triple $m=\left(k,\left\llcorner n_{i} \cdot \varphi_{r}\right\lrcorner, l\right)$ represents

Australasian Journal of Logic (13:5) 2016, Article no. 3 
a set of sort $k$ informally written as $x=\left\{n_{i} \mid \varphi_{r}\left(n_{i}, l\right)\right\}$. Further we will interpret $t \in x$ as $\varphi_{r}(t, l)$ being true via $\operatorname{Tr}_{k}$. Thus, $S t(k, m)$ means that $m$ represents a set of sort $k$.

Next we define arithmetical formulas $\operatorname{Set}_{k}, k=1,2, \ldots$

$\operatorname{Set}_{k}(m) \leftrightharpoons S t(\bar{k}, m)$.

$\operatorname{Set}_{k}(m)$ also means that $m$ represents a set of sort $k$. The difference between $S t(k, m)$ and $\operatorname{Set}_{k}(m)$ is that in the first formula $k$ is a variable of the language $P A T r$ and in the second formula $k$ is an external natural number.

Next we define interpretation $\theta$ of the theory $S A$ in the theory $P A T r$.

Interpretation $t^{\theta}$ of term $t$ is defined by induction on the complexity of $t$.

- Interpretation of variables: $n_{i}^{\theta}=n_{(0, i)}, x_{i}^{(k) \theta}=n_{(k, i)}, k \geqslant 1$.

- If $t=\tau+r$, then $t^{\theta}=\tau^{\theta}+r^{\theta}$.

- If $t=\tau \cdot r$, then $t^{\theta}=\tau^{\theta} \cdot r^{\theta}$.

For each formula $\varphi$ of $S A$ we define its interpretation $\theta(\varphi)$ by induction on the complexity of $\varphi$.

$$
\begin{aligned}
& \theta(t=\tau) \leftrightharpoons t^{\theta}=\tau^{\theta} . \\
& \theta\left(t \in_{k} x^{(k)}\right) \leftrightharpoons \exists m, i, l\left[x^{\theta}=\left(\bar{k},\left\llcorner n_{i} \cdot \varphi_{m}, l\right\lrcorner\right) \wedge \operatorname{Tr}_{k}\left(m, \operatorname{subst}\left(l, i, t^{\theta}\right)\right)\right], k \geqslant 1 . \\
& \theta(\perp) \leftrightharpoons \perp \\
& \theta(\psi \diamond \chi) \leftrightharpoons \theta(\psi) \diamond \theta(\chi) . \\
& \theta(Q n \psi) \leftrightharpoons Q n^{\theta} \theta(\psi) . \\
& \theta\left(\forall x^{(k)} \psi\right) \leftrightharpoons \forall x^{\theta}\left[\operatorname{Set}_{k}\left(x^{\theta}\right) \supset \theta(\psi)\right], k \geqslant 1 . \\
& \theta\left(\exists x^{(k)} \psi\right) \leftrightharpoons \exists x^{\theta}\left[\operatorname{Set}_{k}\left(x^{\theta}\right) \wedge \theta(\psi)\right], k \geqslant 1 .
\end{aligned}
$$

Clearly, if $\varphi$ is a formula of $S A_{s}$, then $\theta(\varphi)$ is a formula of $\operatorname{PATr}_{s}(s \geqslant 0)$.

Theorem 4.1. Suppose $s \geqslant 0$.

1. For any arithmetical formula $\varphi$ with all parameters in the list $n_{1}, \ldots, n_{m}$ :

$$
\operatorname{PATr}_{s} \vdash \theta(\varphi) \equiv \varphi\left[n_{1}, \ldots, n_{m} / n_{1}^{\theta}, \ldots, n_{m}^{\theta}\right] .
$$

2. If $S A_{s} \vdash \varphi$, then $P A T r_{s} \vdash \theta(\overline{\bar{\varphi}})$.

3. $S A_{s+1} \vdash P v_{S A_{s}}(m) \supset P v_{P A T r_{s}}\left(\left\llcorner\theta\left(\overline{\overline{\varphi_{m}}}\right)\right\lrcorner\right)$.

Proof. 1. Proof is by induction on the complexity of $\varphi$.

2. Both $P A T r_{0}$ and $S A_{0}$ are the same as the first-order arithmetic $P A$.

For $s \geqslant 1$ proof is by induction on the length of derivation of $\varphi$. Since logical connectives are preserved in this interpretation, the statement holds for the induction axiom and the classical predicate logic. Peano axioms are the same in both theories. It remains to check the comprehension and choice axioms.

Australasian Journal of Logic (13:5) 2016, Article no. 3 
Suppose $\varphi$ is the comprehension axiom:

$$
\exists z^{(k)} \forall n_{\gamma}\left(n_{\gamma} \in_{k} z \equiv \psi\right) .
$$

Here $k \leqslant s ; \psi$ is a $k$-simple formula with all its parameters in the list $\tilde{x}, \tilde{n}, n_{\gamma}$, where $\tilde{x}$ is a list of set variables $x_{\alpha_{1}}^{\left(k_{1}\right)}, \ldots, x_{\alpha_{p}}^{\left(k_{p}\right)}$ (all different from $z^{(k)}$ ) and $\tilde{n}$ is a list of numerical variables $n_{\beta_{1}}, \ldots, n_{\beta_{v}}$ (all different from $n_{\gamma}$ ).

We need to prove in $P A T r_{s}$ the interpretation of $\overline{\bar{\varphi}}$, which is equivalent to:

$$
\begin{aligned}
\forall n_{\beta_{1}}^{\theta}, \ldots, n_{\beta_{v}}^{\theta} \forall x_{\alpha_{1}}^{\left(k_{1}\right) \theta}, \ldots, x_{\alpha_{p}}^{\left(k_{p}\right) \theta} & \left\{\bigwedge_{j=1}^{p} \operatorname{Set}_{k_{j}}\left(x_{\alpha_{j}}^{\theta}\right)\right. \\
& \left.\supset \exists z^{\theta}\left[\operatorname{Set}_{k}\left(z^{\theta}\right) \wedge \forall n_{\gamma}^{\theta}\left(\theta\left(n_{\gamma} \in z\right) \equiv \theta(\psi)\right)\right]\right\} .
\end{aligned}
$$

Let us fix $n_{\beta_{1}}^{\theta}, \ldots, n_{\beta_{v}}^{\theta}, x_{\alpha_{1}}^{\left(k_{1}\right) \theta}, \ldots, x_{\alpha_{p}}^{\left(k_{p}\right) \theta}$ and assume $\bigwedge_{j=1}^{p} \operatorname{Set}_{k_{j}}\left(x_{\alpha_{j}}^{\theta}\right)$. Then each $x_{\alpha_{j}}^{\theta}(j=1,2, \ldots, p)$ has the form:

$$
x_{\alpha_{j}}^{\theta}=\left(\overline{k_{j}},\left\llcorner n_{\gamma_{j}} . \psi_{j}\right\lrcorner, q_{j}\right),
$$

where $\operatorname{Form}\left(\overline{k_{j}-1},\left\llcorner\psi_{j}\right\lrcorner\right)$ and $E v\left(\left\llcorner\forall n_{\gamma_{j}} \psi_{j}\right\lrcorner, q_{j}\right)$. Every $\psi_{j}$ is a formula of $P A T r_{k-1}$, since $k_{j} \leqslant k$.

We obtain a formula $\psi^{\prime}$ from $\psi$ by changing each numerical subterm $t$ to $t^{\theta}$ and then each atomic subformula $t^{\theta} \in x_{\alpha_{j}}^{\left(k_{j}\right)}$ to the formula $\psi_{j}$, in which $n_{\gamma_{j}}$ is replaced with $t^{\theta}$ and all other parameters are replaced with corresponding values from the evaluation $q_{j}$. Thus, $\psi^{\prime}$ is a formula of $P A T r_{k-1}$ with all its parameters in the list $n_{\gamma}^{\theta}, \tilde{n}^{\theta}$.

Fix an evaluation $l$ of all parameters of $\forall n_{\gamma}^{\theta} \psi^{\prime}$ such that:

$$
(l)_{i}= \begin{cases}n_{\beta_{j}}^{\theta} & \text { if } i=\left(0, \beta_{j}\right), j=1,2, \ldots, v, \\ 0 & \text { otherwise. }\end{cases}
$$

Let us take $r=\left(\bar{k},\left\llcorner n_{\gamma}^{\theta} \cdot \psi^{\prime}\right\lrcorner, l\right)$. We will use $r$ as the value for $z^{(k) \theta}$. We have $\operatorname{Set}_{k}(r)$, and it remains to prove:

$$
\forall n_{\gamma}^{\theta}\left\{\theta\left(n_{\gamma} \in z\right)\left[z^{\theta} / r\right] \equiv \theta(\psi)\right\} .
$$

By the definition of the interpretation $\theta$ we have:

$$
\forall n_{\gamma}^{\theta}\left\{\theta\left(n_{\gamma} \in z^{(k)}\right)\left[z^{\theta} / r\right] \equiv \operatorname{Tr}_{k}\left(\left\llcorner\psi^{\prime}\right\lrcorner, \operatorname{subst}\left(l,(0, \gamma), n_{\gamma}^{\theta}\right)\right)\right\} .
$$

Since $\psi^{\prime}$ is a formula of $P A T r_{k-1}$, by Lemma 2.1.1) we have for any $n_{\gamma}^{\theta}$ :

$$
\begin{aligned}
\operatorname{Tr}_{k}\left(\left\llcorner\psi^{\prime}\right\lrcorner, \operatorname{subst}(l,(0, \gamma)\right. & \left.\left., n_{\gamma}^{\theta}\right)\right) \\
& \equiv \psi^{\prime}\left[n_{\gamma}^{\theta}, n_{\beta_{1}}^{\theta}, \ldots, n_{\beta_{v}}^{\theta} / n_{\gamma}^{\theta},(l)_{\left(0, \beta_{1}\right)}, \ldots,(l)_{\left(0, \beta_{v}\right)}\right] \equiv \psi^{\prime}
\end{aligned}
$$

Australasian Journal of Logic (13:5) 2016, Article no. 3 
by the definition of $l$. So

$$
\forall n_{\gamma}^{\theta}\left[\operatorname{Tr}_{k}\left(\left\llcorner\psi^{\prime}\right\lrcorner, \operatorname{subst}\left(l,(0, \gamma), n_{\gamma}^{\theta}\right)\right) \equiv \psi^{\prime}\right] .
$$

By (1) and (2), it is sufficient to prove:

$$
\forall n_{\gamma}^{\theta}\left[\psi^{\prime} \equiv \theta(\psi)\right] .
$$

Proof of (3)

We prove (3) by induction on the complexity of $\psi$.

Case 1: $\psi$ is $t=\tau$.

Then $\psi^{\prime} \equiv\left(t^{\theta}=\tau^{\theta}\right) \equiv \theta(\psi)$.

Case 2: $\psi$ is $t \in x_{\alpha_{j}}^{\left(k_{j}\right)}$.

Then $x_{\alpha_{j}}^{\theta}$ has the form: $x_{\alpha_{j}}^{\theta}=\left(\overline{k_{j}},\left\llcorner n_{\gamma_{j}} \cdot \psi_{j}\right\lrcorner, q_{j}\right)$ and $\psi^{\prime}$ is the formula $\psi_{j}$, where $n_{\gamma_{j}}$ is replaced with $t^{\theta}$ and all other parameters are replaced with corresponding values from the evaluation $q_{j}$. So

$$
\theta(\psi) \equiv \operatorname{Tr}_{k_{j}}\left(\left\llcorner\psi_{j}\right\lrcorner, \operatorname{subst}\left(q_{j}, \gamma_{j}, t^{\theta}\right)\right) \equiv \psi^{\prime}
$$

by Lemma 2.1.1) and the definition of $\psi^{\prime}$.

Case 3: $\psi$ is $\chi \diamond \eta$. Then

$$
\psi^{\prime} \equiv \chi^{\prime} \diamond \eta^{\prime} \equiv \theta(\chi) \diamond \theta(\eta) \equiv \theta(\psi)
$$

by the inductive assumption.

Case 4: $\psi$ is $Q n_{i} \chi$. Then

$$
\psi^{\prime} \equiv Q n_{i}^{\theta} \chi^{\prime} \equiv Q n_{i}^{\theta} \theta(\chi) \equiv \theta(\psi)
$$

by the inductive assumption.

Since $\psi$ is a $k$-simple formula, it contains no quantifiers over set variables. This completes the proof for the case of the comprehension axiom.

Next we consider the case when $\varphi$ is the choice axiom:

$$
\forall m \exists ! z^{(k)} \psi(m, z) \supset \exists y^{(k+1)} \forall m \exists z^{(k)}[\psi(m, z) \wedge \forall j(j \in z \equiv(m, j) \in y)],
$$

where $k+1 \leqslant s$ and $\psi$ is a $k$-simple formula with all its parameters in the list $\tilde{x}, \tilde{n}, m, z^{(k)}$. As before, $\tilde{x}$ denotes a list of set variables $x_{\alpha_{1}}^{\left(k_{1}\right)}, \ldots, x_{\alpha_{p}}^{\left(k_{p}\right)}$ (all different from $z^{(k)}$ ) and $\tilde{n}$ denotes a list of numerical variables $n_{\beta_{1}}, \ldots, n_{\beta_{v}}$ (all different from $m$ ).

Denote:

$$
\chi\left(n_{\gamma}\right) \leftrightharpoons \exists m^{\theta}, j^{\theta}, z^{(k) \theta}\left[n_{\gamma}=\left(m^{\theta}, j^{\theta}\right) \wedge \operatorname{Set}_{k}\left(z^{\theta}\right) \wedge \theta(\psi(m, z)) \wedge \theta(j \in z)\right],
$$

where $n_{\gamma}$ is a new variable.

Australasian Journal of Logic (13:5) 2016, Article no. 3 
Since $\psi$ is a $k$-simple formula of $S A_{s}$, then it is a $k$-simple formula of $S A_{k}$, so $\theta(\psi)$ is a formula of $P A T r_{k}$ and $\chi$ is a formula of $P A T r_{k}$ with all its parameters in the list $n_{\gamma}, \tilde{n}^{\theta}, \tilde{x}^{\theta}$.

We need to prove in $P A T r_{s}$ the interpretation of $\overline{\bar{\varphi}}$, which is equivalent to:

$$
\begin{aligned}
& \forall n_{\beta_{1}}^{\theta}, \ldots, n_{\beta_{v}}^{\theta} \forall x_{\alpha_{1}}^{\left(k_{1}\right) \theta}, \ldots, x_{\alpha_{p}}^{\left(k_{p}\right) \theta}\left\{\bigwedge_{j=1}^{p} \operatorname{Set}_{k_{j}}\left(x_{\alpha_{j}}^{\theta}\right)\right. \\
& \wedge \forall m^{\theta} \exists ! z^{(k) \theta}\left[\operatorname{Set}_{k}\left(z^{\theta}\right) \wedge \theta(\psi(m, z))\right] \supset \exists y^{(k+1) \theta}\left\{\operatorname{Set}_{k+1}\left(y^{\theta}\right)\right. \\
& \left.\left.\wedge \forall m^{\theta} \exists z^{(k) \theta}\left[\operatorname{Set}_{k}\left(z^{\theta}\right) \wedge \theta(\psi(m, z)) \wedge \forall j^{\theta}[\theta(j \in z) \equiv \theta((m, j) \in y)]\right]\right\}\right\} .
\end{aligned}
$$

Let us fix $n_{\beta_{1}}^{\theta}, \ldots, n_{\beta_{v}}^{\theta}, x_{\alpha_{1}}^{\left(k_{1}\right) \theta}, \ldots, x_{\alpha_{p}}^{\left(k_{p}\right) \theta}$ and an evaluation $l$ of parameters of the formula $\forall n_{\gamma} \chi$ such that:

$$
(l)_{i}= \begin{cases}n_{\beta_{j}}^{\theta} & \text { if } i=\left(0, \beta_{j}\right), j=1,2, \ldots, v \\ x_{\alpha_{j}}^{\theta} & \text { if } i=\left(k_{j}, \alpha_{j}\right), j=1,2, \ldots, p \\ 0 & \text { otherwise. }\end{cases}
$$

Assume the premises of (4) and take $r=\left(\overline{k+1},\left\llcorner n_{\gamma} \cdot \chi\right\lrcorner, l\right)$. We will use $r$ as the value for $y^{(k+1) \theta}$. Since $\chi$ is a formula of $P A T r_{k}$ and $E v\left(\left\llcorner\forall n_{\gamma} \chi\right\lrcorner, l\right)$, we have $\operatorname{Set}_{k+1}(r)$. It remains to prove:

$$
\begin{aligned}
\forall m^{\theta} \exists z^{(k) \theta}\left\{\operatorname{Set}_{k}\left(z^{\theta}\right) \wedge \theta(\right. & (m, z)) \\
& \left.\wedge \forall j^{\theta}\left\{\theta(j \in z) \equiv \theta\left((m, j) \in y^{(k+1)}\right)\left[y^{\theta} / r\right]\right\}\right\} .
\end{aligned}
$$

By the definition of the interpretation $\theta$ we have for any $m^{\theta}, j^{\theta}$ :

$$
\begin{gathered}
\theta\left((m, j) \in y^{(k+1)}\right)\left[y^{\theta} / r\right] \\
\left.\equiv \operatorname{Tr}_{k+1}\left(\llcorner\chi\lrcorner, \operatorname{subst}\left(l, \gamma,\left(m^{\theta}, j^{\theta}\right)\right)\right)[\text { by Lemma 2.1 } 1)\right] \equiv \\
\chi\left[n_{\gamma}, n_{\beta_{1}}^{\theta}, \ldots, n_{\beta_{v}}^{\theta}, x_{\alpha_{1}}^{\theta}, \ldots, x_{\alpha_{p}}^{\theta} /\left(m^{\theta}, j^{\theta}\right),(l)_{\left(0, \beta_{1}\right)}, \ldots,(l)_{\left(0, \beta_{v}\right)},(l)_{\left(k_{1}, \alpha_{1}\right)},\right. \\
\left.\ldots,(l)_{\left(k_{p}, \alpha_{p}\right)}\right] \equiv[\text { by the definition of } l] \equiv \chi\left(\left(m^{\theta}, j^{\theta}\right)\right) \\
\quad \equiv \exists z^{(k) \theta}\left[\operatorname{Set}_{k}\left(z^{\theta}\right) \wedge \theta(\psi(m, z)) \wedge \theta(j \in z)\right] .
\end{gathered}
$$

Therefore for any $m^{\theta}, j^{\theta}$ :

$$
\theta\left((m, j) \in y^{(k+1)}\right)\left[y^{\theta} / r\right] \equiv \exists z^{(k) \theta}\left[\operatorname{Set}_{k}\left(z^{\theta}\right) \wedge \theta(\psi(m, z)) \wedge \theta(j \in z)\right] .
$$

Now we will prove (5). Let us fix $m$. From the premises there exists a unique $u^{(k) \theta}$ such that

$$
\operatorname{Set}_{k}\left(u^{\theta}\right) \wedge \theta(\psi(m, u))
$$

Australasian Journal of Logic (13:5) 2016, Article no. 3 
It remains to prove that for any $j^{\theta}$ :

$$
\theta(j \in u) \equiv \theta\left((m, j) \in y^{(k+1)}\right)\left[y^{\theta} / r\right] .
$$

Proof of (8)

$\Rightarrow$. This part follows immediately from (6) and (7).

$\Leftarrow$. Suppose $\theta\left((m, j) \in y^{(k+1)}\right)\left[y^{\theta} / r\right]$. Then by (6) there exists $z^{(k) \theta}$ such that $\operatorname{Set}_{k}\left(z^{\theta}\right) \wedge \theta(\varphi(m, z)) \wedge \theta(j \in z)$. By the uniqueness of $u$ we have $z^{\theta}=u^{\theta}$ and $\theta(j \in u)$.

3. This is proven by formalizing the proof of part 2 in $S A_{s+1}$.

Corollary 4.2. If $S A \vdash \varphi$, then $\operatorname{PATr} \vdash \theta(\overline{\bar{\varphi}})$.

Proof. Follows from Theorem 4.1.2).

Corollary 4.3. Suppose $s \geqslant 0$.

1. If $S A R_{s} \vdash \varphi$, then $\operatorname{PATr}_{s} \vdash \theta(\overline{\bar{\varphi}})$.

2. $S A R_{s+1} \vdash P v_{S A R_{s}}(m) \supset \operatorname{Pv}_{P A T r_{s}}\left(\left\llcorner\theta\left(\overline{\overline{\varphi_{m}}}\right)\right\lrcorner\right)$.

Proof. 1. The proof is the same as for Theorem 4.1.2). Alternatively, it follows from Theorem 4.1.2) because $S A R_{s}$ is a subsystem of $S A_{s}$.

2. This is proven by formalizing the proof of part 1 in $S A R_{s+1}$.

In [2] we constructed an interpretation $\varphi^{\sim}$ of $P A T r$ in $S A$ and proved the following theorem.

Theorem 4.4. 1. For an arithmetical formula $\varphi, \varphi^{\sim}$ is the same as $\varphi$.

2. For $s \geqslant 0$ : if $\operatorname{PATr}_{s} \vdash \varphi$, then $S A_{s} \vdash(\overline{\bar{\varphi}})^{\sim}$.

Corollary 4.5. If PATr $\vdash \varphi$, then $S A \vdash(\overline{\bar{\varphi}})^{\sim}$.

Thus, due to corollaries 4.2 and 4.5, the theories $S A$ and PATr are mutually interpretable.

\section{Comparing the Proof-theoretical Strengths of Fragments}

Corollaries 4.2 and 4.5 imply that the theories $S A$ and $P A T r$ are equiconsistent. Theorems 4.1.2) and 4.4.2) imply that fragments $S A_{s}$ and $P A T r_{s}$ are equiconsistent $(s \geqslant 0)$.

Lemma 5.1. For $s \geqslant 0$ :

1. $P A T r_{s+1} \vdash P v_{P A T r_{s}}(m) \wedge E v(m, l) \supset \operatorname{Tr}_{s+1}(m, l)$.

2. $\operatorname{PATr}_{s+1} \vdash \operatorname{Con}_{P A T r_{s}}$.

3. $P A T r_{s+1} \vdash \operatorname{Con}_{S A_{s}}$.

Australasian Journal of Logic (13:5) 2016, Article no. 3 
Proof. 1. Proof is by induction on the length of derivation of $\varphi_{m}$ using Lemma 2.1.2).

2. We describe derivation in $P A T r_{s+1}$ informally.

Assume $P v_{P A T r_{s}}(\llcorner\perp\lrcorner)$. Then by part $1, \operatorname{Tr}_{s+1}(\llcorner\perp\lrcorner, l)$ for the empty evaluation $l$. This contradicts the axiom $(\operatorname{Tr} 4)$.

So $\neg \operatorname{Pv}_{P A T r_{s}}(\llcorner\perp\lrcorner)$, that is $C_{P A T r_{s}}$.

3. We describe derivation in $P A T r_{s+1}$ informally. Assume $P v_{S A_{s}}(\llcorner\perp\lrcorner)$. Then by Theorem 4.1.3), we get $P v_{P A T r_{s}}(\llcorner\perp\lrcorner)$, which contradicts part 2 .

Theorem 5.2. For $s \geqslant 0, S A_{s+1} \vdash \operatorname{Con}_{S A_{s}}$.

Proof. By Lemma 5.1.3), PATr $r_{s+1} \vdash \operatorname{Con}_{S A_{s}}$. Since $\operatorname{Con}_{S A_{s}}$ is a closed arithmetical formula, we have by Theorem 4.4, $S A_{s+1} \vdash \operatorname{Con}_{S A_{s}}$.

This theorem shows that each fragment $S A_{s}$ is weaker than next fragment $S A_{s+1}(s \geqslant 0)$. The same is true for fragments of PATr (Lemma 5.1.2).

\section{DisCussion}

In this paper we described the axiomatic theories $S A$ and $S A R$ that can have applications in reverse mathematics. These theories are versions of second order arithmetic with countably many sorts for sets of natural numbers. Their multi-sorted language allows to state some mathematical statements in more natural form than in the language of second order arithmetic but this requires more research. We studied metamathematical properties of the theories $S A$ and $S A R$. In particular, we showed that $S A$ is mutually interpretable with the theory of arithmetical truth PATr introduced in [1]. We showed that each fragment $S A_{s}$ is proof-theoretically weaker than next fragment $S A_{s+1}$.

In [4] Simpson used model theory to compare different subsystems of second order arithmetic. Here we study metamathematical properties of the theories using the interpretation technique. Unlike the model method, the interpretation technique may be adjusted to intuitionistic versions of the axiomatic theories, which can be used in constructive reverse mathematics.

Next we plan to investigate further the metamathematical properties of $S A R$; some of the proofs for $S A$ do not apply to $S A R$ because of its restricted induction axiom. We also plan to develop some parts of reverse mathematics with respect to the theories $S A, S A R$ and their fragments, and some parts of constructive reverse mathematics with respect to intuitionistic versions of these theories. 


\section{ACKNOWLEDGEMENTS}

The author is grateful to the editor and referee for valuable comments and suggestions that helped to improve this paper.

\section{REFERENCES}

[1] F. Kachapova. Interpretation of constructive multi-typed theory in the theory of arithmetical truth. Lobachevskii Journal of Mathematics, 36(4):332-341, 2015. URL: http: //link.springer.com/article/10.1134\%2FS1995080215040034

[2] F. Kachapova. Metamathematical properties of a constructive multi-typed theory. To be published.

[3] E. Mendelson. Introduction to Mathematical Logic. Chapman and Hall/CRC, Boca Raton, Florida, 2009.

[4] S.G. Simpson. Subsystems of Second Order Arithmetic. Cambridge University Press, 2010.

Auckland University of Technology, Auckland, New Zealand

E-mail address: farida.kachapova@aut.ac.nz

Australasian Journal of Logic (13:5) 2016, Article no. 3 